Article

\title{
Accumulation Behaviors of Different Particles and Effects on the Breakdown Properties of Mineral Oil under DC Voltage
}

\author{
Min Dan ${ }^{1,2}$, Jian Hao ${ }^{1, *}$, Ruijin Liao ${ }^{1}$, Lin Cheng ${ }^{3,4}$, Jie Zhang ${ }^{3,4}$ and Fei Li ${ }^{3,4}$ \\ 1 State Key Laboratory of Power Transmission Equipment \& System Security and New Technology, \\ Chongqing University, Chongqing 400044, China; danmin@cqu.edu.cn (M.D.); rjliao@cqu.edu.cn (R.L.) \\ 2 State Grid Chongqing Nanan Power Supply Company, Chongqing 401223, China \\ 3 Najing NARI Group Corporation, State Grid Electric Power Research Institute, Nanjing 211000, China; \\ chenglin@sgepri.sgcc.com.cn (L.C.); zhangjie3@sgepri.sgcc.com.cn (J.Z.); lifei6@sgepri.sgcc.com.cn (F.L.) \\ 4 Wuhan NARI Co. Ltd., State Grid Electric Power Research Institute, Wuhan 430077, China \\ * Correspondence: haojian2016@cqu.edu.cn; Tel.: +86-182-2301-0926
}

Received: 19 May 2019; Accepted: 12 June 2019; Published: 16 June 2019

\begin{abstract}
Particles in transformer oil are harmful to the operation of transformers, which can lead to the occurrence of partial discharge and even breakdown. More and more researchers are becoming interested in investigating the effects of particles on the performance of insulation oil. In this paper, a simulation method is provided to explore the motion mechanism and accumulation characteristics of different particles. This is utilized to explain the effects of particle properties on the breakdown strength of mineral oil. Experiments on particle accumulation under DC voltage as well as DC breakdown were carried out. The simulation results are in agreement with the experimental results. Having a DC electrical field with a sufficient accumulation time and initial concentration are advantageous for particle accumulation. Properties of impurities determine the bridge shape, conductivity characteristics, and variation law of DC breakdown voltages. Metal particles and mixed particles play more significant roles in the increase of current and electrical field distortion. It is noteworthy that cellulose particles along with metal particles cannot have superposition influences on changing conductivity characteristics and the electrical field distortion of mineral oil. The range of electrical field distortion is enlarged as the particle concentration increases. Changes in the electrical field distribution and an increase in conductivity collectively affect the DC breakdown strength of mineral oil.
\end{abstract}

Keywords: mineral oil; different particles; accumulation behavior; breakdown voltage; DC voltage

\section{Introduction}

In order to meet the urgent demand for energy delivery, China has vigorously developed large-capacity and long-distance ultra-high voltage transmission technology over the last 10 years. As a result, the market for large transformers has enlarged, which has strengthened the requirement for large volume and high-quality transformers. Mineral oil-paper insulation is widely used in large transformers, and this determines the safety, stability, and insulation performance of transformers. Transformer damage is mainly caused by insulation problems, among which particle pollution of insulating oil is a significant factor [1].

A large body of literature has expounded sources of particles in power equipment. For power transformers, metal and non-metal particles are the main components of solid particles. Metal particles mainly include copper and iron particles, while non-metallic particles are mainly made up of carbon particles and cellulose particles. Cellulose impurities are the main part of non-metallic impurities, 
accounting for more than $90 \%$ of these impurities [2-4]. Owing to the process of production, assembly, transportation, and incomplete oil filtering, impurities may be left in oil [5-9]. Meanwhile, impurities may be caused by the aging of oil-paper insulation, partial discharge, and part wearing due to long-term operation [5-9]. A few particles move to the joint position between different components with the oil flow. Others gather in the region where the flow rate is slower to form a path connecting conductors, which brings a severe challenge to the stable operation of transformers as well as leading to partial discharge or breakdown [10-12].

The CIGRE Working Group 12.17 conducted a statistical analysis of the fault causes of twenty-two transformers and forty bushings $(765 \mathrm{kV})$ and pointed out that the source and hazard of particles must be paid attention to for transformers $400 \mathrm{kV}$ and above [6]. In recent years, in view of the influence of particles on the insulation performance of oil, scholars at home and abroad have carried out research on the movement characteristics of particles in mineral oil as well as the influence of impurity bridges on insulation performance. Mahmud S. et al. studied the bridging phenomena of cellulose particles in mineral oil based on spherical electrodes and needle-plate electrodes under different DC and AC voltages. It was found that DC voltage was the main factor in bridge formation [13-17]. Yuan Li et al. studied the generation and development of cellulose bridges and their influences on partial discharge under DC voltage [18]. In [19,20], a millimeter-diameter metal particle's movement properties and its movement were studied using a theoretical model. In [21,22], the motion trajectory of a metal particle in flowing oil under AC voltage was provided. The movement process of a millimeter-diameter metal particle between electrodes has been investigated under various forms of voltage, and it has been stated that the voltage form and particle concentration have important impacts on the motion characteristics of metal particles [23,24]. The movement properties of millimeter-diameter metal particles under AC voltage have been investigated by numerous researchers. Nevertheless, studies of the motion characteristics of smaller metal particles and their DC breakdown features are lacking. The bridging processes of non-metallic and metal impurities has scarcely been researched. In addition, most simulation models used to analyze the impact of particles on the breakdown performance of insulating oil are still being devoted to the study of electrical field distortion of insulating oil caused by large spherical particles, and comprehensive analyses on the influence of current characteristics along with changes in the electrical field owing to particle accumulation in insulation oil are lacking.

In this paper, experiments on the motion characteristics of cellulose particles, copper particles, and mixed particles are carried out under DC voltage. In addition, DC breakdown voltages of mineral oil contaminated by different concentration particles are measured. A simulation model of particle accumulation is built through Comsol software. The computation model can be utilized to explain the differences in the motion and accumulation properties of different particles in mineral oil. Moreover, the variation in the DC breakdown strength of mineral oil containing different particles with different concentration is analyzed.

\section{Simulation and Experiment}

\subsection{Motion Model of Metal Particles}

Assuming that insulating oil cannot be compressed, suspended metal particles in oil withstand gravity and buoyancy in the vertical direction. In the horizontal direction, forces include the dielectrophoretic force $\left(F_{D E P 1}\right)$ [25], the viscous drag force $\left(F_{d r a g 1}\right)$ [25], and the Coulomb force $\left(F_{C 1}\right)$ [25]. Based on the microstructure of metal particles in Figure 1a, the dielectrophoretic force of spherical metal particles with the radius $r$ can be calculated:

$$
F_{D E P 1}=2 \pi \varepsilon_{m} r^{3} \frac{\delta_{p}-\delta_{m}}{\delta_{p}+2 \delta_{m}} \nabla|E|^{2}
$$

where $\varepsilon_{m}$ represents the permittivity of insulation oil, and $\sigma_{m}$ and $\sigma_{p}$ stand for the conductivity of insulation liquid and the metal particle respectively. 


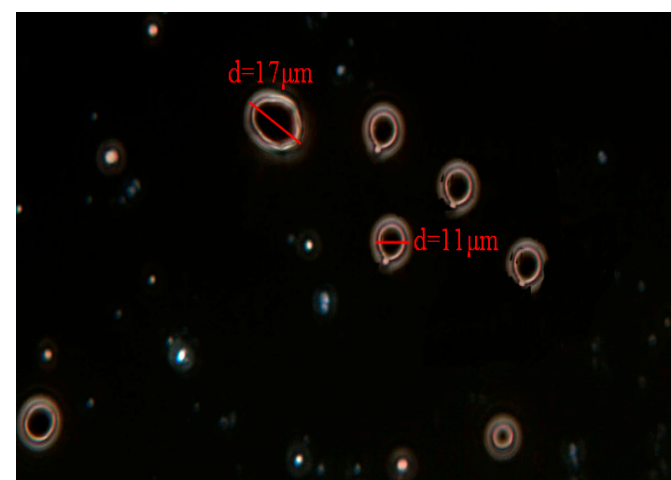

(a) metal particles

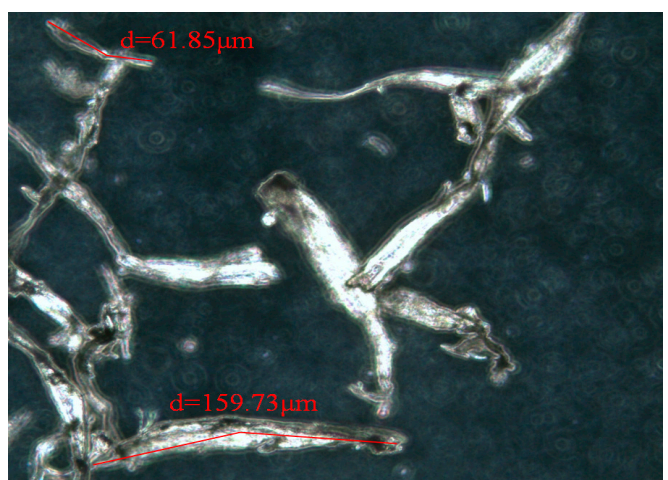

(b) cellulose particles

Figure 1. The microstructures of metal particles (a) and cellulose particles (b).

Viscous drag force $\left(F_{\text {drag } 1}\right)$ acts on the metal particle, as shown in Formula (2). Because of the nonlinearity of the viscous drag force, the correction factor $\left(C_{d}\right)$ related to the Reynolds coefficient $(\mathrm{Re})$ should be used to correct the viscous drag force.

$$
\begin{gathered}
F_{\text {drag } 1}=6 \pi \eta r v_{p 1} C_{d} \\
C_{d}= \begin{cases}0.17+0.72 \ln (\operatorname{Re}) & \operatorname{Re} \geq 2 \\
1 & \operatorname{Re}<2\end{cases} \\
\operatorname{Re}=\frac{2 r \rho_{m}\left|v_{p 1}\right|}{\eta}
\end{gathered}
$$

where $\eta$ and $\rho_{m}$ are the viscosity and density of the insulation liquid, and $v_{p 1}$ represents the velocity of the metal particle.

When the metal particle with the radius of $r$ collides with one electrode, a certain amount of charge $\left(q_{1}\right)$ is obtained, as calculated by Formula (5). Then, plugging Formula (5) into (6) yields the following expression:

$$
\begin{gathered}
q_{1}=\frac{2}{3} \pi^{3} \varepsilon_{m} \varepsilon_{0} r^{2} E \\
F_{C 1}=0.83 q_{1} E=0.553 \pi^{3} E^{2} r^{2} \varepsilon_{m} \varepsilon_{0}
\end{gathered}
$$

Owing to the dynamic model of metal particles represented by Equations (1) to (6), the velocity of the metal particle can be calculated by Formula (7):

$$
v_{p 1}=\frac{r^{2} \varepsilon_{m}\left(\delta_{p}-\delta_{m}\right)}{3 \eta\left(\delta_{p}+2 \delta_{m}\right)} \nabla E^{2}
$$

\subsection{Motion Model of Cellulose Particles}

As a result of the difference in micromorphology between metal particles and cellulose particles, their kinetic models are different. The micromorphology of cellulose particles is illustrated in Figure $1 \mathrm{~b}$. It is assumed that cellulose particles are regarded as ellipsoids to simplify the computational investigation. The kinetic model of cellulose particles is as follows:

$$
\begin{gathered}
F_{D E P 2}=\frac{\pi \varepsilon_{m} \varepsilon_{0}}{12} d^{2} l\left[\frac{\alpha}{\alpha-1}-f(\beta)\right]^{-1} \nabla E^{2} \\
\alpha=\frac{\varepsilon_{p}}{\varepsilon_{m}} \\
f(\beta)=\xi\left[\left(1-\xi^{2}\right) \operatorname{coth}^{-1} \xi+\xi\right]
\end{gathered}
$$




$$
\begin{gathered}
\xi=\beta\left(\beta^{2}-1\right)^{-1 / 2} \\
\beta=l / d \\
F_{d r a g 2}=3 \pi \eta d v_{p 2} g(\beta) \\
g(\beta)=\frac{8}{3}\left[\frac{-2 \beta}{\beta^{2}-1}+\frac{2 \beta^{2}-1}{\left(\beta^{2}-1\right)^{3 / 2}} \ln \frac{\beta+\left(\beta^{2}-1\right)^{1 / 2}}{\beta-\left(\beta^{2}-1\right)^{1 / 2}}\right]^{-1} \\
F_{C 2}=0.553 \pi^{3} E^{2} r^{2} \varepsilon_{m} \varepsilon_{0}
\end{gathered}
$$

where $\varepsilon_{m}$ and $\varepsilon_{p}$ represent the permittivity of insulation oil and cellulose impurities, and $d$ and $l$ stand for the diameter and length of the cellulose particle. Using Formulas (8)-(15), the velocity of the cellulose particle $v_{p_{2}}$ can be attained using

$$
v_{p 2}=\lim _{\beta \rightarrow \infty} \frac{\varepsilon_{m} \varepsilon_{0}}{24 \eta} l^{2} \frac{\ln 2 \beta-0.5}{\ln 2 \beta-1} \nabla E^{2}
$$

\subsection{Accumulation Model of Particles}

Based on the results of the impurity accumulation experiments, the velocity of the particles $\left(v_{p}\right)$ is closely related to the concentration $(c)$. There is a certain particle concentration $\left(c_{c r i t}\right)$ that prevents particle movement, because a large number of particles are bound to form bridges [26]. Thus, the relationship between the velocity and concentration of particles is as follows [26]:

$$
v_{p}^{\prime}= \begin{cases}0 & c \geq c_{c r i t} \\ v_{p}-v_{p} \frac{c}{c_{c r i t}} & c<c_{c r i t}\end{cases}
$$

Fick's diffusion law, which contains the velocity of particles, is utilized to calculate the dynamic behavior of particle concentration. Because of no chemical reaction takes place, the reaction rate expression for species $R_{i}$ is equal to 0 . The flux vector (or molar flux) $N$ is associated with the Fick equation and is used under boundary conditions and for flux computation. $u_{m}\left(10^{-7} \mathrm{~s}^{*} \mathrm{~mol} / \mathrm{kg}\right)$ is the charge mobility, where $c$ is the concentration of impurities, and $D\left(10^{-11} \mathrm{~m}^{2} / \mathrm{s}\right)$ is seen as the diffusion parameter:

$$
\begin{aligned}
& \frac{\partial c_{i}}{\partial t}+\nabla \bullet N_{i}=R_{i} \\
& N_{i}=-D_{i} \nabla c_{i}-z_{i} u_{m, i} F c_{i} \nabla V
\end{aligned}
$$

The different physics interface involving only the scalar electric potential can be interpreted in terms of the charge relaxation process. The basic equation is Ohm's law:

$$
\begin{aligned}
& \boldsymbol{J}=\delta \boldsymbol{E}+\boldsymbol{J}_{\boldsymbol{e}} \\
& \delta=\delta_{p}^{2.3 c} * \delta_{o i l}^{1-2.3 c}
\end{aligned}
$$

where $J_{e}$ is an externally generated current density, and $J_{e}=0 . \sigma$ is the collective conductivity of insulation oil and impurities, which is derived according to the Looyenga Formula (31). The static form of the equation of current continuity then reads

$$
\nabla \bullet J=0=-\nabla \bullet\left(\delta \nabla V-J_{e}\right)=-\nabla \bullet(\delta \nabla V)=0
$$

Then, according to the fundamental equation of electrostatic field, the electric field distribution can be illustrated by

$$
\begin{aligned}
& E=-\nabla V \\
& \nabla^{2} V=0
\end{aligned}
$$


Based on motion models and accumulation model of particles, the results of the accumulation concentration together with the electrical field distribution variation for cellulose particles, metal particles, as well as mixed particles can be obtained.

\subsection{Particle Accumulation and Oil Breakdown Measurement}

A standard oil cup containing a sphere-sphere electrode made of copper material with a diameter of $13 \mathrm{~mm}$ was used in the experiments. In accordance with IEC 60156, an electrode distance of $2.5 \mathrm{~mm}$ was used in the DC breakdown experiments. To study the accumulation characteristics of the three kinds of particles, $7.5 \mathrm{~mm}$ and $12.5 \mathrm{kV}$ were utilized. The oil cup was situated under a digital camera which recorded the process of particle accumulation. The current was measured by a Keithley electrometer (6517B). The experimental setup for the DC breakdown voltage testing is shown in Figure 2. HCDJC-100kV/5kVA was utilized to provide high DC voltage. The signal together with data was controlled and attained by the computer system.

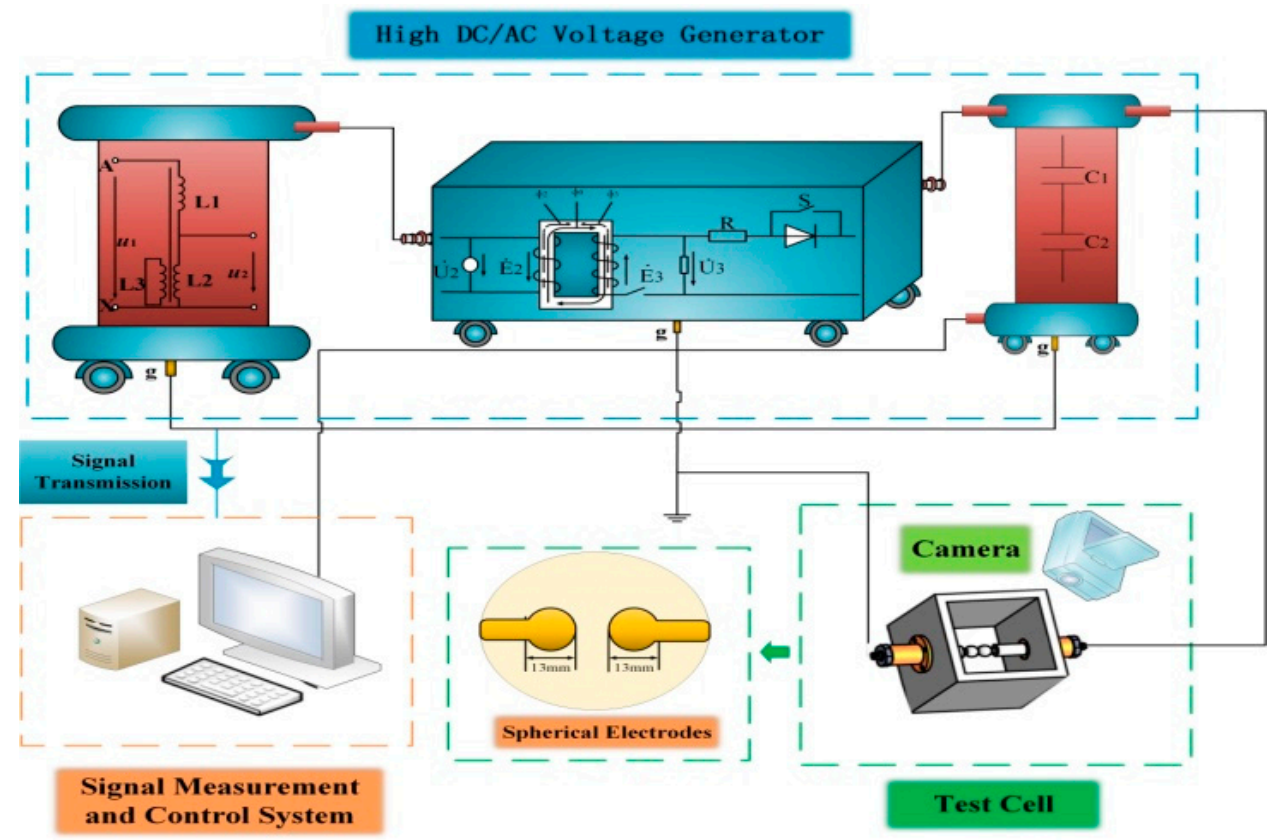

Figure 2. The experimental platform for the particle accumulation and DC breakdown test.

Three kinds of impurities were used in all experiments: cellulose particles, metal particles, and their mixture. Cellulose particles were produced by rubbing new insulation paper through metal files of different sizes. In this paper, cellulose particles with sizes of 63-150 $\mu \mathrm{m}$ and 150-250 $\mu \mathrm{m}$ were prepared. Spherical copper particles with $15 \mu \mathrm{m}$ diameters came from Beijing Hongyu New Materials Co., Ltd. (Beijing, China). The contamination levels of particles can be seen in Table 1. Before the experiments, all samples were dried for $48 \mathrm{~h}$ using a vacuum box at $90{ }^{\circ} \mathrm{C}$ and $133 \mathrm{~Pa}$. The parameters of clean mineral oil can be seen in Table 2. For the sake of simplicity, abbreviations are used in Table 3 to stand for different samples. After homogeneous mixing of particles and mineral oil, the samples were sealed in the vacuum box for $24 \mathrm{~h}$.

Each particle accumulation experiment lasted $1500 \mathrm{~s}$. To ensure even particle distribution, a stirrer was used to stir samples. Moreover, every sample was repeatedly broken down eight to ten times. The average value was treated as the final DC breakdown voltage for this sample. All tests were performed at room temperature. 
Table 1. Parameters of mineral oil.

\begin{tabular}{cccccc}
\hline \multicolumn{5}{c}{ Contamination Level } \\
\hline Cellulose & $0.001 \%$ & $0.003 \%$ & $0.006 \%$ & $0.009 \%$ & $0.012 \%$ \\
particles & $0.1 \mathrm{~g} / \mathrm{L}$ & $0.3 \mathrm{~g} / \mathrm{L}$ & $0.6 \mathrm{~g} / \mathrm{L}$ & $1 \mathrm{~g} / \mathrm{L}$ & $1.5 \mathrm{~g} / \mathrm{L}$ \\
Metal particles & $0.003 \%+0.1 \mathrm{~g} / \mathrm{L}$ & $0.003 \%+0.3 \mathrm{~g} / \mathrm{L}$ & $0.003 \%+0.6 \mathrm{~g} / \mathrm{L}$ & $0.003 \%+1 \mathrm{~g} / \mathrm{L}$ & - \\
Mixed particles & $0.012 \%+0.1 \mathrm{~g} / \mathrm{L}$ & $0.012 \%+0.3 \mathrm{~g} / \mathrm{L}$ & $0.012 \%+0.6 \mathrm{~g} / \mathrm{L}$ & $0.012 \%+1 \mathrm{~g} / \mathrm{L}$ & - \\
\hline
\end{tabular}

Table 2. Parameters of mineral oil.

\begin{tabular}{cc}
\hline Parameters & Mineral Oil \\
\hline Density in $\mathrm{g} / \mathrm{cm}^{3}$ & 0.89 \\
Dynamic viscosity in $\mathrm{mm}^{2} / \mathrm{s}\left(20^{\circ} \mathrm{C}\right)$ & 25.70 \\
Permittivity $\left(20^{\circ} \mathrm{C}, 50 \mathrm{~Hz}\right)$ & 2.20 \\
Volume resistivity in $\Omega \cdot \mathrm{m}\left(20^{\circ}\right)$ & $4.68 \times 10^{13}$ \\
\hline
\end{tabular}

Table 3. Abbreviations of samples analyzed in the experiments.

\begin{tabular}{cc}
\hline Samples & Sample Composition \\
\hline DMCP & Dry mineral oil + cellulose particles \\
DMMP & Dry mineral oil + metal particles \\
DMCM & Dry mineral oil + mixed particles of cellulose and metal particles \\
MO & Pure mineral oil \\
\hline
\end{tabular}

\section{Experimental Results and Discussion}

\subsection{Particle Accumulation Simulation Results}

The parameters of the particle accumulation simulation model are described in Table 4 . Three-dimensional simulation diagrams of the accumulation of cellulose particles, metal particles, and mixed particles in insulation oil at $10 \mathrm{~s}$ and $600 \mathrm{~s}$ are shown in Figure 3 . With an increase in computational time, impurity accumulation between electrodes was more evident. Samples containing cellulose particles formed initial filamentous bridges faster than those only containing metal particles at the start stage of voltage application. At $600 \mathrm{~s}$, the concentration of mixed particles was the largest, followed by cellulose particles and metal particles. The concentrations of cellulose particles and copper particles were, respectively, 1.2 and 3.7 times less than that of mixed particles.

Table 4. Parameters of the particle accumulation model.

\begin{tabular}{ccc}
\hline Parameters & Cellulose Particles & Metal Particles \\
\hline Density, $\mathrm{g} / \mathrm{cm}^{3}\left(20{ }^{\circ} \mathrm{C}\right)$ & 1.2 & 8.6 \\
Permittivity $\left(20^{\circ} \mathrm{C}, 50 \mathrm{~Hz}\right)$ & 4.4 & $10^{5}$ \\
Size, $\mu \mathrm{m}$ & $63-150$ & 15 \\
Volume resistivity, $\Omega \cdot \mathrm{m}\left(20^{\circ} \mathrm{C}\right)$ & $2 \times 10^{6}$ & $2 \times 10^{-8}$ \\
Initial concentration $\left(c_{0}\right), \mathrm{mol} / \mathrm{m}^{3}$ & 0.0005 & 0.0005 \\
DC voltage, $\mathrm{kV}$ & 11.25 & 11.25 \\
Distance, $\mathrm{mm}$ & 7.5 & 7.5 \\
\hline
\end{tabular}




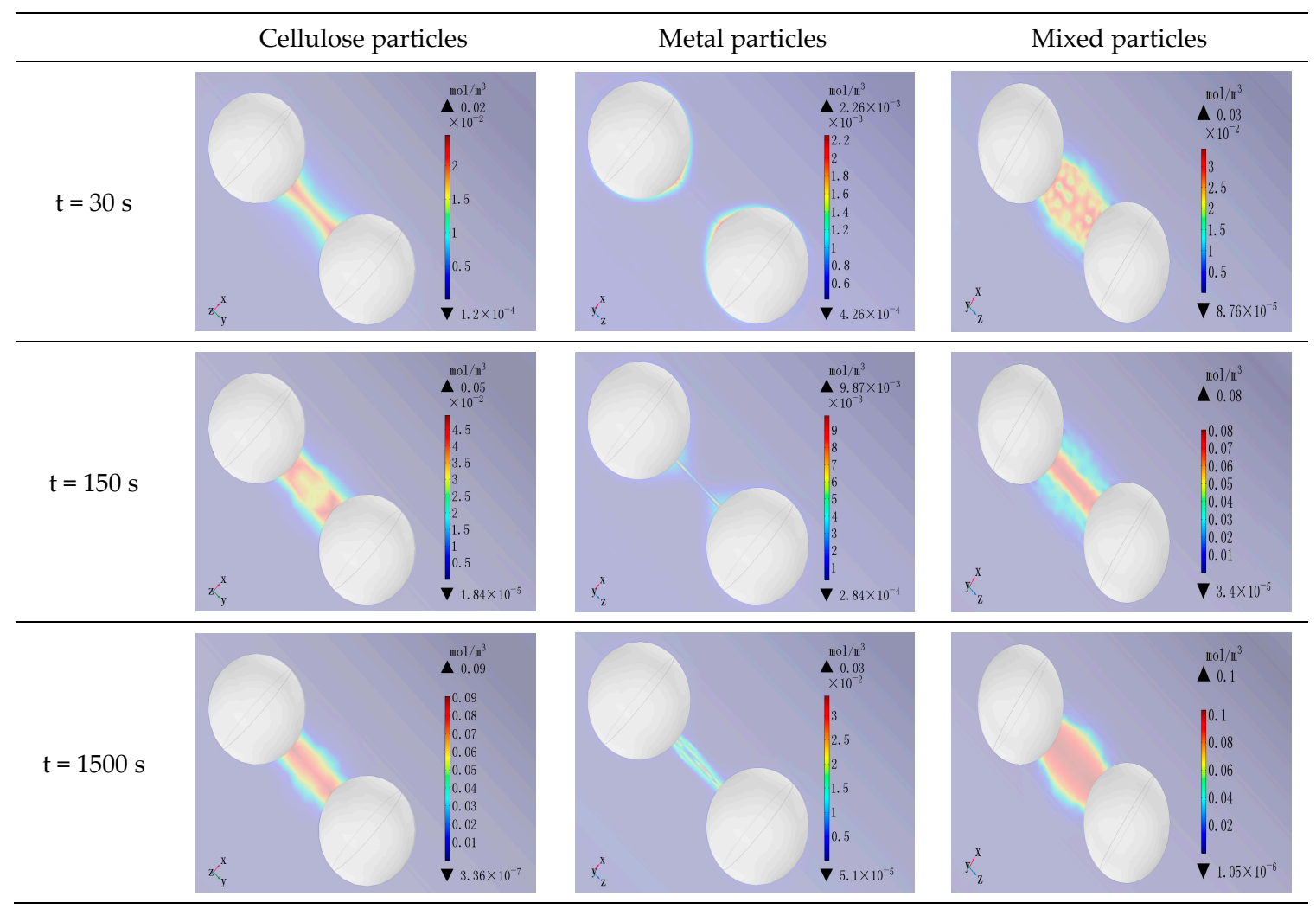

Figure 3. Simulation results of different kinds of particle accumulation in mineral oil at different times.

\subsection{Experimental Results of Particle Accumulation.}

Figure 4 illustrates the dynamic behavior of particle accumulation under DC voltage, in which the concentration levels of cellulose particles, metal particles, and mixed particles were respectively $0.009 \%$ by weight, $0.1 \mathrm{~g} / \mathrm{L}$, and the sum of both. The distance and DC voltage between the spherical electrodes were $7.5 \mathrm{~mm}$ and $11.25 \mathrm{kV}$, respectively. The left electrode was a cathode, and the right one was an anode.

\begin{tabular}{|c|c|c|c|}
\hline Samples & $30 \mathrm{~s}$ & $150 \mathrm{~s}$ & $1500 \mathrm{~s}$ \\
\hline DMCP & & & \\
\hline DMMP & & & \\
\hline DMCM & & & \\
\hline
\end{tabular}

Figure 4. Particle accumulation status under the same DC voltage in mineral oil at different time. 
It is obvious that impurity bridges form for the three kinds of particles, as shown in Figure 4. The non-charged particles move toward the higher electrical field area under the attraction of dielectrophoresis, i.e., the central position of the electrode. The particles are charged on the side of the adsorbed electrode. When the Coulomb force of the particles are enough to resist the dielectrophoresis force, the particle moves uniformly to the opposite electrode under combined forces including the Coulomb force, the viscous drag force, and dielectrophoresis force. A few of the particles move back and forth between the electrodes according to the above rule. It is noteworthy that large particles are more likely to move rapidly, because the electrical field force and dielectrophoresis force of the particle are proportional to the square of a particle's radius, and the viscous drag force is proportional to the particle size. However, as a result of lacking enough charge to resist the dielectrophoresis attraction in a certain time period, the rest of particles adhere to electrodes which form a tip on the surface of electrodes. The tip may enhance the local electrical field, attract surrounding particles to move there, and continuously lengthen the particle chain. The initial filamentous bridge is not completely parallel to the electrical field line, which results in parallel movement of the particle bridge under the action of force in the horizontal direction and merges with other small bridges; thus, its thickness increases.

As shown in Figure 4 (sample DMCP) a thin fiber bridge was observed after $30 \mathrm{~s}$ in mineral oil containing cellulose particles. After that, the thickness of cellulose bridges increased gradually. The complete bridge formed until approximately $600 \mathrm{~s}$. Then, notable changes in the cellulose bridge were not seen. For mineral oil contaminated by metal particles, most metal particles sunk down, owing to their larger densities. A filamentous bridge formed at about $500 \mathrm{~s}$ (Figure 4, sample DMMP), which was so unstable that it migrated back and forth between electrodes, which may be attributed to the impact of the strong electrical field. The ultimate metal bridge appeared until $1200 \mathrm{~s}$. As for mixed particles (Figure 4, sample DMCP), the complete bridge formed more quickly than those for single impurities. Furthermore, it was observed that the complete bridge was densest when the transverse force among thin bridges made up of mixed particles was larger. It can be inferred that the simulation model depicting the changing course of particles aggregation is in agreement with the actual process. Thus, the simulation model can accurately describe the impact of impurities on motion as well as on the accumulation characteristics of particles. Different attributes of particles determined the difference in the trajectory. The trajectories of cellulose particles and metal particles under DC electrical field observed from Figure 4 is shown in Figure 5.

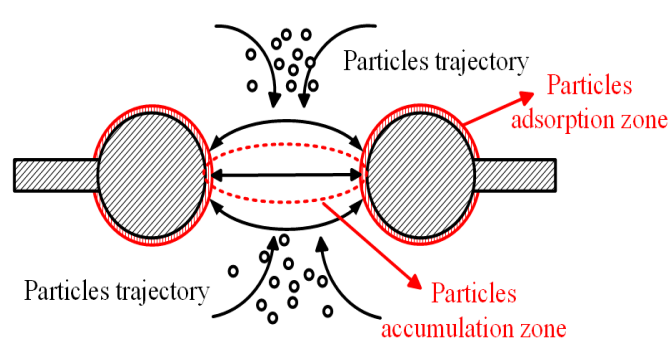

(a) cellulose particles

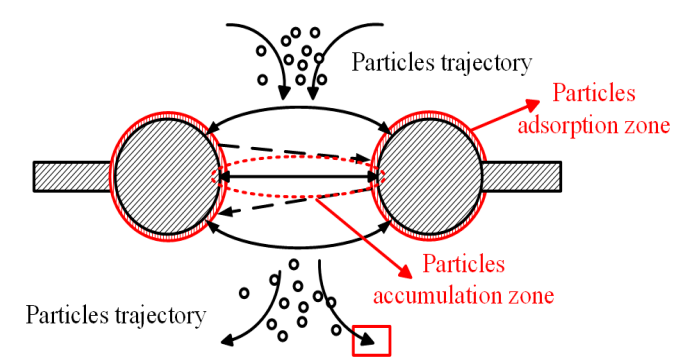

(b) metal particles

Figure 5. Motion trajectory of cellulose particles (a) and metal particles (b) under DC voltage in mineral oil.

\subsection{Effect of Particles Accumulation on Oil DC Breakdown Voltage}

The Weibull distribution model was utilized to analyze the DC breakdown voltages of samples. Its mathematical expression is shown by Equation (22), where $t$ stands for the DC breakdown strength; $\alpha$ stands for the scale parameter, which describes the characteristic breakdown strength of oil; and $\beta$ represents the shape parameter, which reflects the changing rate of breakdown probability with an increase in DC voltage. The data samples used in this study were complete, so the empirical distribution function $F_{\mathrm{n}}\left(t_{\mathrm{i}}\right)$ could be calculated by Formula (23), where $i$ represents the order of test samples, and $n$ is the number of samples. Figure 6 is the Weibull probability distribution plot of 
different samples under the DC breakdown voltage. With an increase in the concentration of particles, the Weibull curve moved to the left. Namely, the average breakdown values of samples reduced as the particle concentration increased. The relationship between the average breakdown voltages of mineral oil containing cellulose particles, metal particles, and mixed particles and the particles concentration are shown in Figure 7. The DC breakdown voltages of oil samples contaminated with metal particles and mixed particles were found to be lower than those of mineral oil containing cellulose particles. In summary, metal impurities and mixed particles have more significant impacts on the DC breakdown characteristics of mineral oil:

$$
\begin{gathered}
F(t ; \alpha, \beta)=1-\exp \left[-\left(\frac{t}{\alpha}\right)^{\beta}\right] \\
F_{n}\left(t_{i}\right)=\frac{i-0.375}{n+0.25} \times 100 \%
\end{gathered}
$$

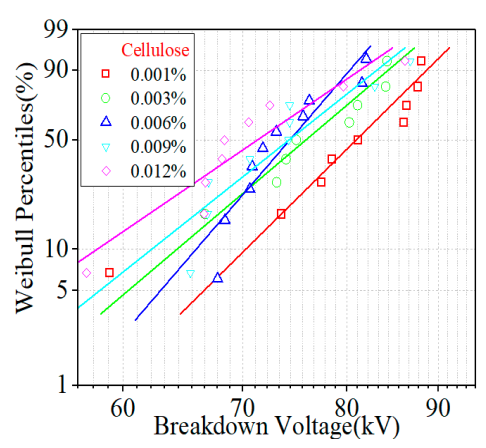

(a) DMCP

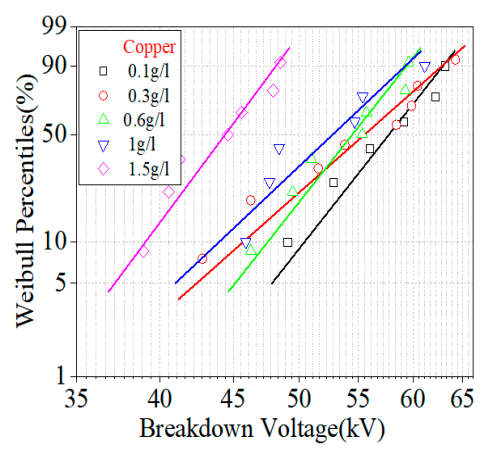

(b) DMMP

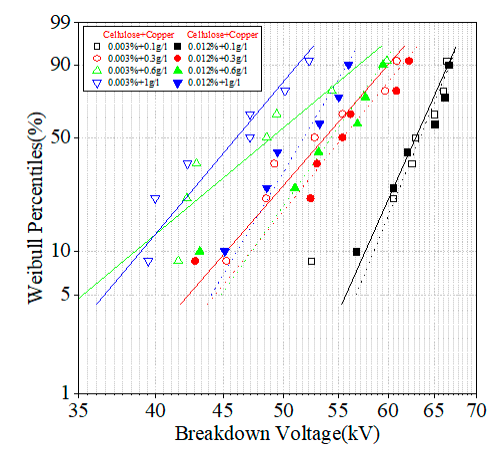

(c) DMCM

Figure 6. Weibull probability distribution plot of DC breakdown voltage for different samples DMCP (a), DMMP (b) and DMCM (c).

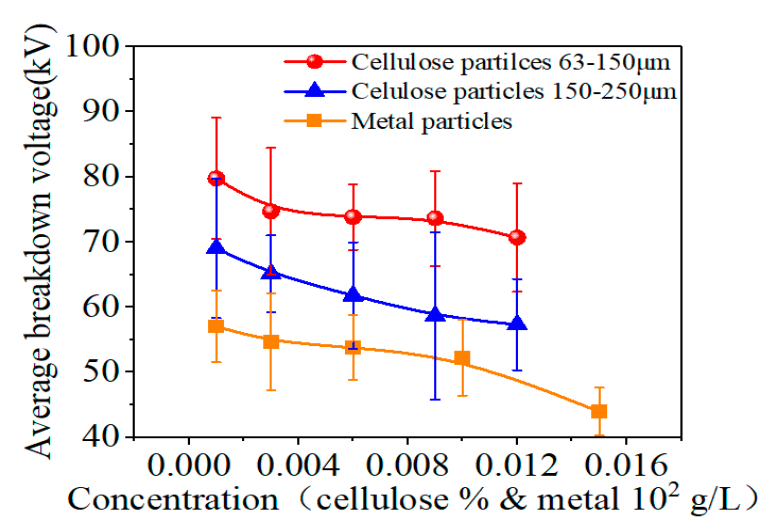

(a) cellulose, metal particles

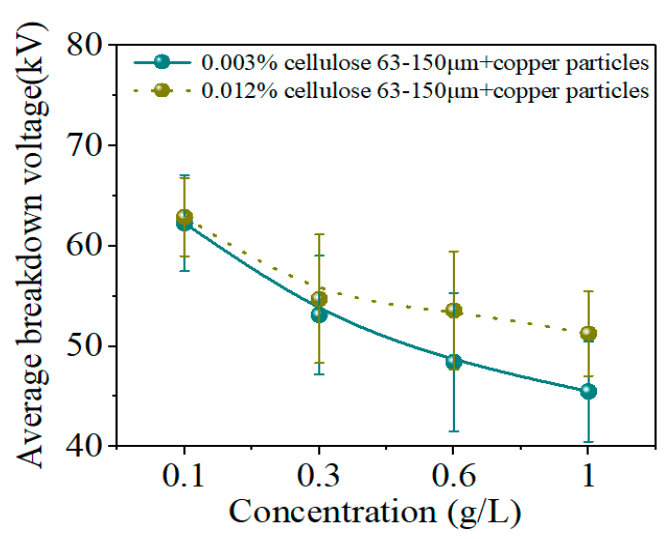

(b) mixed particles

Figure 7. Average DC breakdown voltage of mineral oil with cellulose or metal particles (a), and mixed particles (b).

\subsection{Difference Analysis of the Effects of Different Particles on the Oil DC Breakdown Voltage}

First of all, impurities have a prominent effect on the conductivity of mineral oil. The changing current properties of mineral oil are displayed in Figure 8. Upon the application of DC voltage, instantaneous polarization current appears in both clean oil and contaminated oil. For clean oil, the polarization current decreases to a conduction current. Yet, due to the effect of particles' back and forward motion between electrodes, causing charging and discharging together with formation of bridges, the current in contaminated oil increases little by little until the saturation level is reached. The increasing degree of current saturation in contaminated oil compared to clean oil occurs in 
decreasing order for mixed particles, metal particles, and cellulose particles. The saturated current in mineral oil containing mixed particles is the largest, almost seven times larger than that of clean oil, followed by copper particles ( 5.5 times) and cellulose particles ( 2.8 times). As a result, metal particles or mixed particles more easily cause insulation oil to partially discharge and even break down. Since the sum of the saturated current of cellulose particles and metal particles is not equal to the saturated current of mixed particles, cellulose particles coupled with metal particles cannot have a superposition effect on the conductivity of oil. There is no doubt that the bridge formation of particles under a DC electrical field can significantly improve the conductivity of mineral oil, which is one of main reasons for the decrease in the breakdown strength of oil. Furthermore, there is a corresponding relation between current and particle aggregation state, which is shown in Figure 9.

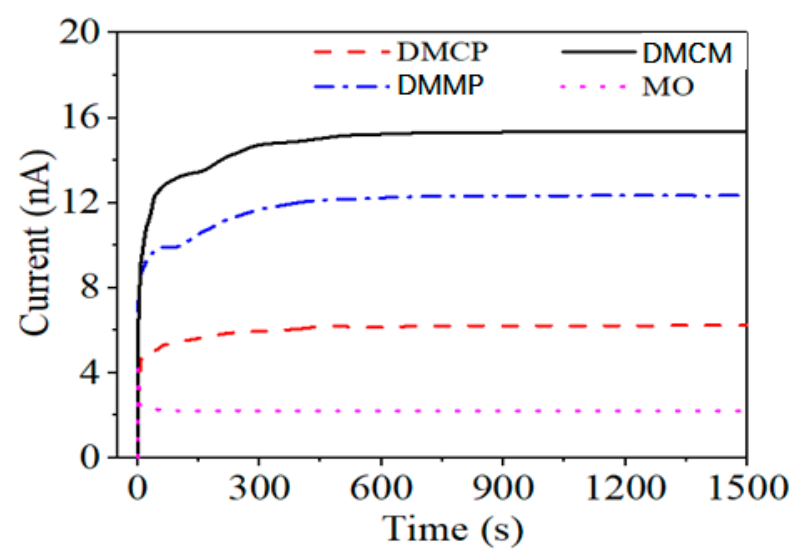

Figure 8. Changes in the current in mineral oil containing different particles under the same DC voltage.

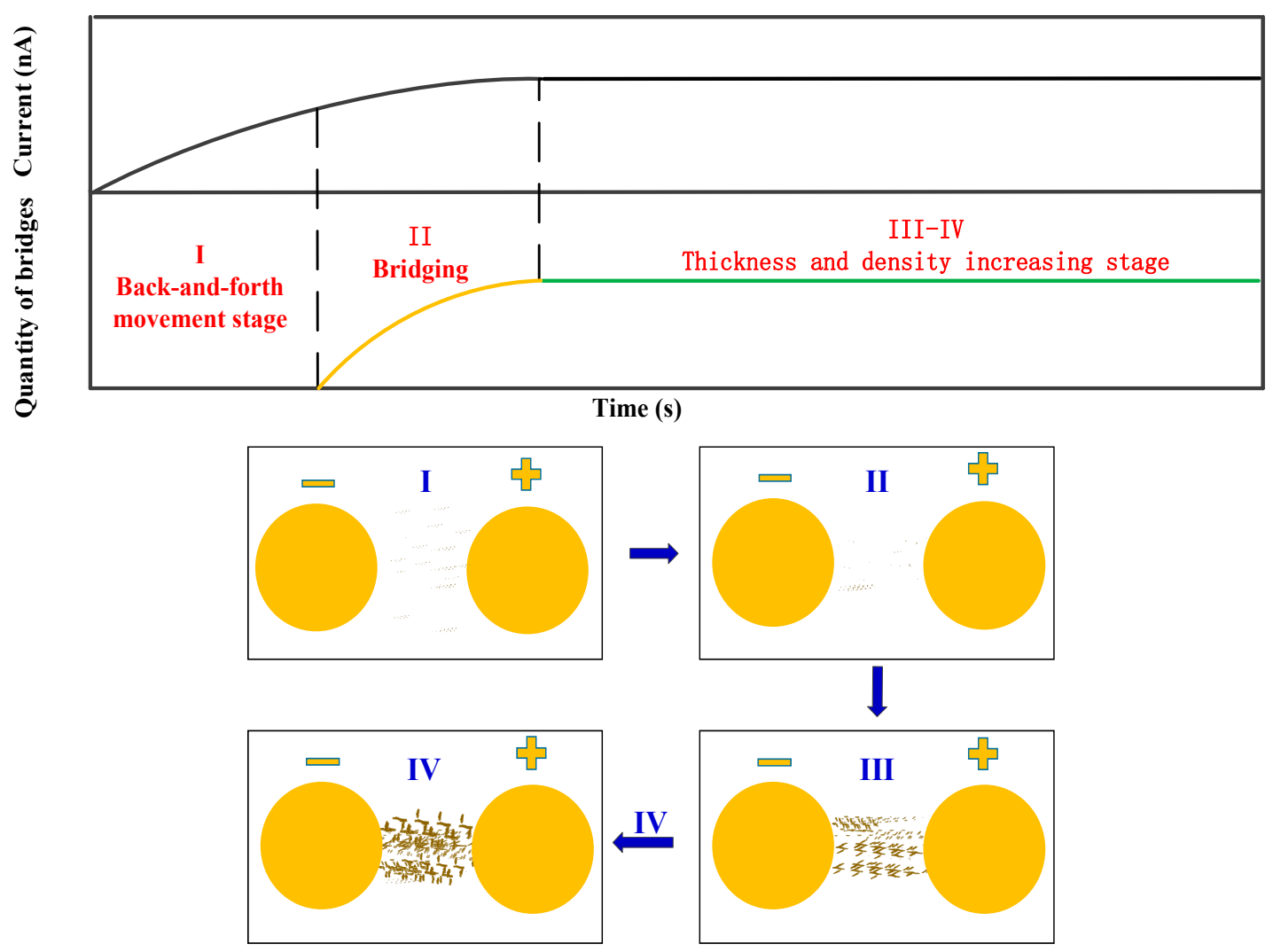

Figure 9. Relationship between oil conductivity current and particle aggregation. 
Then, taking advantage of the effective simulation model shown in Section 3.1, relationships among concentrations, particles properties, and the variation in electrical field strength were analyzed in order to explain the DC breakdown results. Figures 10 and 11 display three dimensional particle accumulation patterns as well as the DC electric field distribution of insulation oil under three different initial concentrations at $600 \mathrm{~s}$. It can be observed that firstly, accumulation degrees increase as the initial concentration increases. Secondly, the range of electrical field distortion caused by particle accumulation is also gradually enlarged.
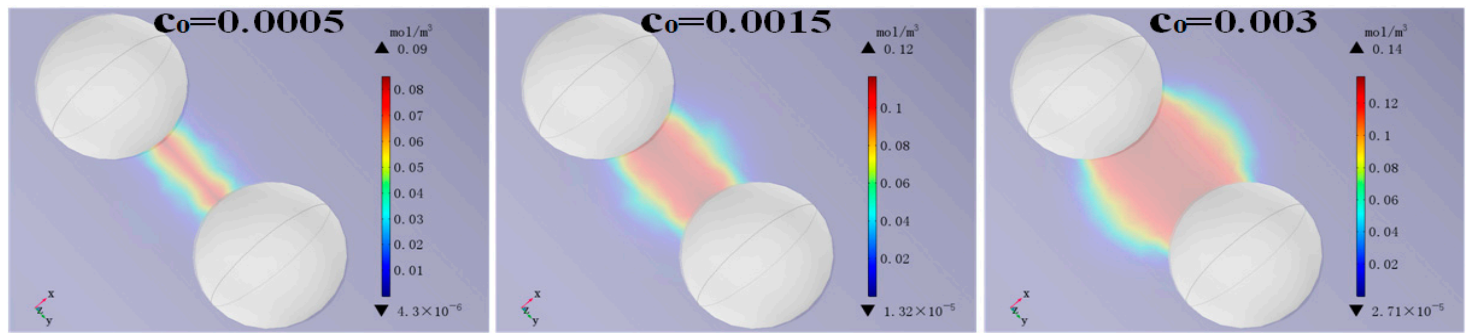

(a) cellulose particles
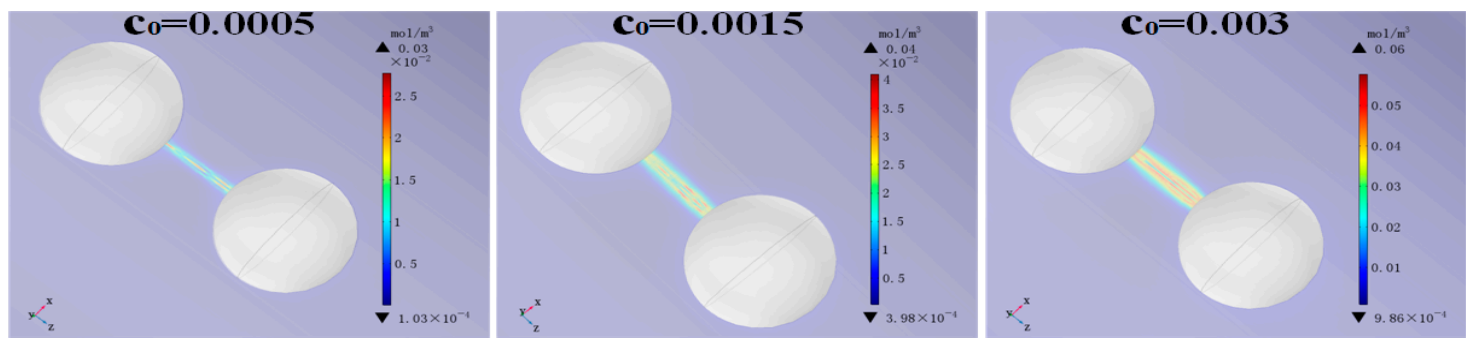

(b) copper particles
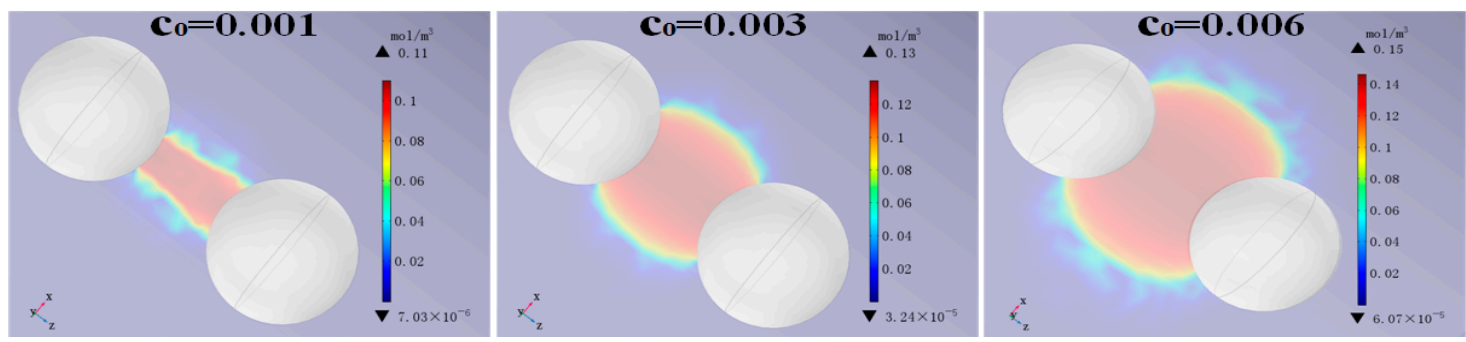

(c) Mixed particles

Figure 10. Particle accumulation patterns under three different initial concentrations for cellulose particles (a), copper particles (b) and mixed particles (c) at $600 \mathrm{~s}$.
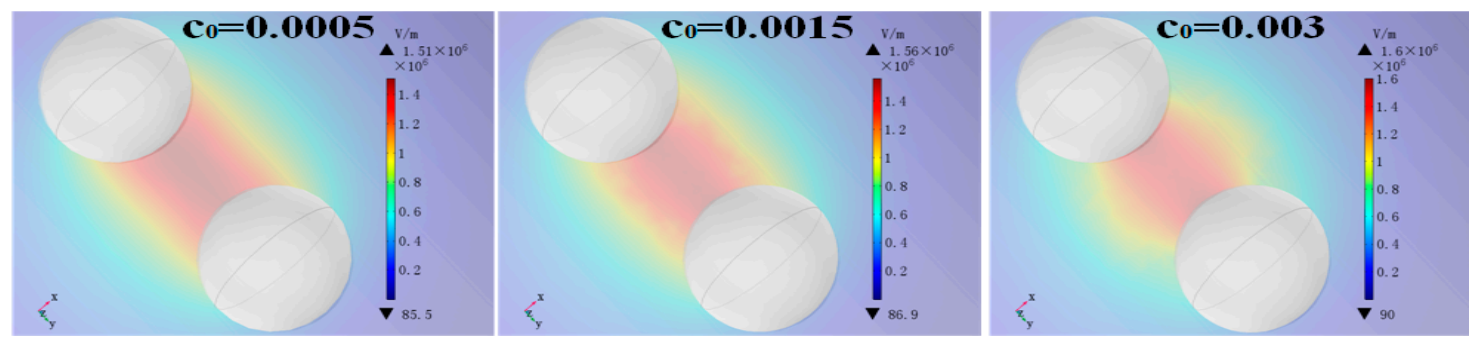

(a) cellulose particles

Figure 11. Cont. 

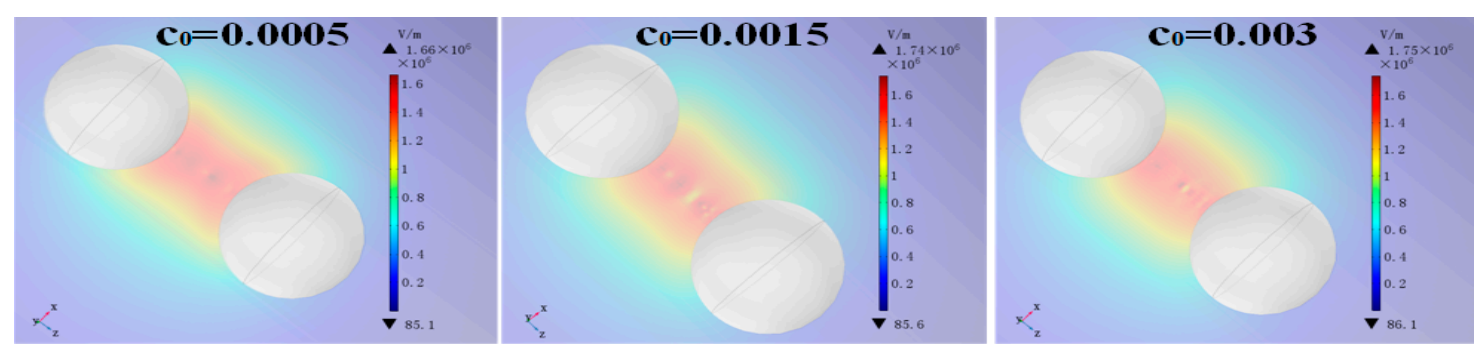

(b) copper particles

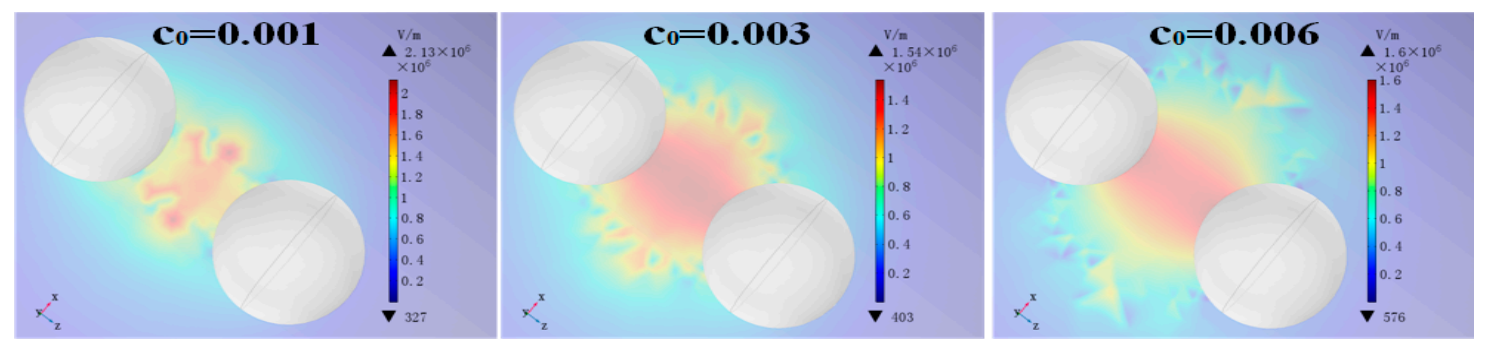

(c) mixed particles

Figure 11. DC electric field distribution of mineral oil contained cellulose particles (a), copper particles (b) and mixed particles (c) under three different initial concentrations at $600 \mathrm{~s}$.

Figure 12 shows the influence of the particle concentration on the maximum electrical field strength $\left(E_{\max }\right)$ of contaminated mineral oil at $600 \mathrm{~s}$. It can be seen that metal particles almost play a more prominent part in electrical field distortion than cellulose particles. Additionally, the saturated current of metal particles is larger than that for cellulose impurities, so the breakdown voltage of mineral oil polluted by metal particles is smaller than that contaminated by cellulose particles. Figures 10 and 11 also imply that cellulose particles as well as metal particles cannot have a superposition effect on the electric field distortion of insulation oil. For mineral oil contaminated by cellulose or metal particles, $E_{\text {max }}$ increases as the particle concentration increases, which indicates that electrical field distortion combined with the conductivity variation leads to degradation of the insulation strength of mineral oil. As for mineral oil containing mixed particles, though electrical field distortion weakens with an increasing concentration, conductivity obviously increases because of the increasing accumulation degree. Hence, changes in the electrical field distribution together with an increase in conductivity collectively affects the DC breakdown characteristics of mineral oil.

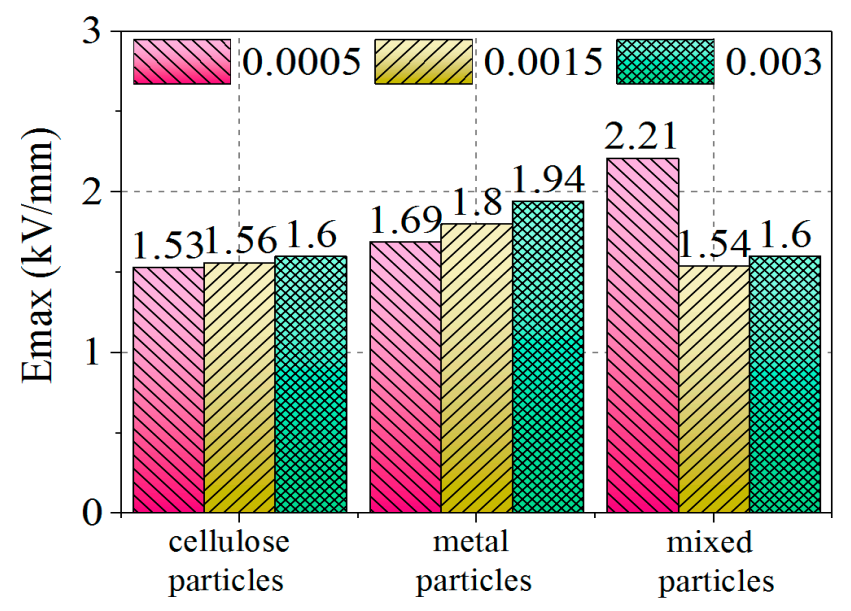

Figure 12. $E_{\max }$ of mineral oil and natural ester containing different particles under three different initial concentrations at $600 \mathrm{~s}$. 
The effect of pressure on breakdown voltage of insulation oil has been investigated, which indicates that the increasing of pressure can increase breakdown voltage of oil [27]. However, few researchers have studied the effects of temperature or pressure effect on particles' accumulation properties. This needs further study in the future.

\section{Conclusions}

In this paper, simulations and experiments were carried out to investigate the accumulation characteristics of cellulose, copper, and mixed particles in mineral oil under DC voltage. The DC breakdown voltages of mineral oil with different particle concentrations were measured. The conclusions obtained from this study are as follows.

The simulation model was able to reveal the process of particle bridging in mineral oil under DC voltage. The simulation results showed that as the experiment duration increased, particle accumulation became more evident. The accumulation concentration of mixed particles was the largest, followed by cellulose particles and metal particles, which is in agreement with the experimental results. In the particle accumulation experiments, it was also obvious that metal particles have difficulty forming stable bridges, while bridges of mixed particles were the densest and thickest among the tested compounds. Moreover, the larger the initial concentration of particles, the more obvious the accumulation phenomenon as well as the electrical field distortion.

The properties of particles determine the accumulation shapes, conductivity characteristics, and variation law of breakdown voltages. The increasing degrees of saturated current in contaminated oil compared to clean oil in decreasing order were found to be mixed particles, metal particles, and cellulose particles. Moreover, metal particles were also shown to play a more prominent part in electrical field distortion than cellulose particles. Therefore, the breakdown voltage of mineral oil contaminated by cellulose particles was larger than that of mineral oil containing copper particles. Nevertheless, it is noteworthy that cellulose particles along with metal particles cannot have a superposition effect on the conductivity characteristics and electrical field distortion of insulation oil.

The test and computational results indicated that changes in the electrical field distribution together with an increase in conductivity collectively affect the breakdown strength of mineral oil. The bridge formation of particles under DC electrical field was shown to significantly improve the conductivity of oil, which is one of main reasons for the decrease in breakdown strength of mineral oil containing particles. The simulation results of the particle accumulation model showed that as the duration of the experiment increased, the range of electrical field distortion caused by particles accumulation gradually expanded, which is also one of main reasons for the decrease in the DC breakdown strength of mineral oil containing particles.

Author Contributions: Designed the experiments, did the motion characteristics experiments and breakdown voltage measurement, wrote the paper, M.D.; improved the simulation method, analyzed the experiment data and revised the paper, J.H.; contributed discussion, R.L., L.C., J.Z., and F.L.

Funding: This research was funded by [National Key R\&D Program of China] grant number [2017YFB0902704], [Science and Technology Project of State Grid Corporation-Research on Insulation Defect Analysis and Testing Technology of UHV Converter Transformer], [Joint Funds of the National Natural Science Foundation of China] grant number [U1866603], [Funds for Innovative Research Groups of China] grant number [51321063].

Conflicts of Interest: The authors declare no conflict of interest.

\section{References}

1. Wang, X.; Wang, Z. Particle effect on breakdown voltage of mineral and ester based transformer oils. In Proceedings of the IEEE Conference on Electrical Insulation and Dielectric Phenomena, Quebec City, QC, Canada, 26-29 October 2008; p. 598.

2. CIGRE. Effect of Particles on Transformer Dielectric Strength; WG 17/SC12; CIGRE: Paris, France, 2000.

3. Lu, W.; Liu, Q. Effect of cellulose particles on impulse breakdown in ester transformer liquids in uniform electric fields. IEEE Trans. Dielectr. Electr. Insul. 2015, 22, 2554-2564. [CrossRef] 
4. Zhao, T. Research of the Effect of Bubbles and Cellulose Particles on Impulse Breakdown in Transformer Oil. Ph.D. Thesis, North China Electric Power University, Beijing, China, 2017.

5. Li, Z.; Ma, Q. Monitoring the particle pollution degree of $500 \mathrm{kV}$ transformer's insulation oil. Transformer 1999, 36, 31-34.

6. Wu, R.; Liu, J.; Chen, J.; Zhang, Z. Analysis of insulating oil particulate pollution in $500 \mathrm{kV}$ transformer and reactor. Inn. Mongo. Electr. Power 2013, 31, 35-37.

7. Xing, L.; Zhang, G. Test and analysis of graininess in 500kV transformer oil. Transformer 2009, 46, 40-43.

8. Wei, L.; Su, Z.; Qi, J. Study on particulate contamination detection of insulating oil for $1000 \mathrm{kV}$ transformer and its influence factor. Insul. Mater. 2015, 6, 50-53.

9. Zhang, G.; Li, X.; Liu, H.; Lu, L. Study on particle size in $500 \mathrm{kV}$ transformer insulation oil. In Proceedings of the Annual Academic Conference of Tianjin Electric Power Society, Tianjin, China, 20-22 October 2008.

10. Dan, M.; Hao, J.; Li, Y.; Liao, R.; Yang, L.; Wang, Q.; Zhang, S. Analysis of bridging phenomenon in mineral oil and natural ester contaminated with cellulose particles under different DC electrical field. In Proceedings of the 20th International Symposium on High Voltage Engineering, Buenos Aires, Argentina, 28 August 2017.

11. Dan, M.; Hao, J.; Qin, W.; Liao, R.; Zou, R.; Zhu, M.; Liang, S. Effect of different impurities on motion characteristics and breakdown properties of insulation oil under DC electrical field. In Proceedings of the 2018 IEEE International Conference on High Voltage Engineering and Application (ICHVE 2018), Athens, Greece, 10-13 September 2018; pp. 1-4.

12. Zhou, Y.; Hao, M.; Chen, G.; Wilson, G.; Jarman, P. Study of the charge dynamics in mineral oil under a non-homogeneous field. IEEE Trans. Dielectr. Electr. Insul. 2015, 22, 2473-2482. [CrossRef]

13. Hao, J.; Liao, R.; Dan, M.; Li, Y.; Li, J.; Liao, Q. Comparative study on the dynamic migration of cellulose particles and its effect on the conductivity in natural ester and mineral oil under DC electrical field. IET Gener. Transm. Distrib. 2017, 11, 2375-2383. [CrossRef]

14. Mahmud, S.; Chen, G.; Golosnoy, I.O.; Wilson, G. Bridging in contaminated transformer oil under AC, DC and DC biased AC electric field. In Proceedings of the 2013 IEEE Electrical Insulation and Dielectric Phenomena, Shenzhen, China, 20-23 October 2013; pp. 943-946.

15. Mahmud, S.; Chen, G.; Golosnoy, I.O.; Wilson, G.; Jarman, P. Experimental studies of influence of DC and AC electric fields on bridging in contaminated transformer oil. IEEE Trans. Dielectr. Electr. Insul. 2015, 22, 152-160. [CrossRef]

16. Mahmud, S.; Chen, G.; Golosnoy, I.O.; Wilson, G.; Jarman, P. Bridging phenomenon in contaminated transformer oil. In Proceedings of the International Conference on Condition Monitoring and Diagnosis, Piscataway, NJ, USA, 23-27 September 2012; pp. 180-183.

17. Li, J.; Zhang, Q.; Li, Y. Generation process of impurity bridges in oil-paper insulation under DC voltage. High Volt. Eng. 2016, 12, 211-218.

18. Li, Y.; Zhang, Q.; Li, J.; Wang, T.; Dong, W.; Ni, H. Study on micro bridge impurities in oil-paper insulation at DC voltage: Their generation, growth and interaction with partial discharge. IEEE Trans. Dielectr. Electr. Insul. 2016, 23, 2213-2222. [CrossRef]

19. Wang, S.; Shi, J.; Li, J. The effect of a macro-particle on the partial property of transformer oil. High Volt. Eng. 1994, 20, 26-29.

20. Fu, S. The acquired charge of macro-particle and its effect on the partial discharge of transformer oil. High Volt. Eng. 2000, 26, 49-50.

21. Tang, J.; Zhu, L.M.; Ma, S.X. Characteristics of suspended and mobile micro bubble partial discharge in insulation oil. High Volt. Eng. 2010, 36, 1341-1346.

22. Ma, S.X.; Tang, J.; Zhang, M.J. Simulation study on distribution and influence factors of metal particles in transaction transformer. High Volt. Eng. 2015, 41, 3628-3634.

23. Wang, Y.Y.; Li, Y.L.; Wei, C.; Zhang, J.; Li, X. Copper particle effect on the breakdown strength of insulating oil at combined AC and DC voltage. J. Electr. Eng. Technol. 2017, 12, 865-873. [CrossRef]

24. Wang, Y.; Li, X. Motion characteristic of copper particle in insulating oil under AC and DC voltages. In Proceedings of the 19th IEEE International Conference on Dielectric Liquid, Manchester, UK, 25-29 June 2017; pp. 25-29.

25. Dan, M.; Hao, J.; Liao, R.; Li, Y.; Yang, L. Different motion and bridging characteristics of fiber particles in mineral oil and natural ester under DC voltage. Power Syst. Technol. 2018, 42, 665-672. 
26. Naciri, N. Finite Element Analysis for Power System Component: Dust Accumulation in Transformer Oil. Ph.D. Thesis, University of Southampton, Southampton, UK, 2011.

27. Butcher, M.; Neuber, A.; Krompholz, H.; Dickens, J. Effect of temperature and pressure on DC pre-breakdown current in transformer oil. In Proceedings of the 31st IEEE International Conference on Plasma Science, Baltimore, MD, USA, 28 June-1 July 2004; pp. 1-4.

(C) 2019 by the authors. Licensee MDPI, Basel, Switzerland. This article is an open access article distributed under the terms and conditions of the Creative Commons Attribution (CC BY) license (http://creativecommons.org/licenses/by/4.0/). 